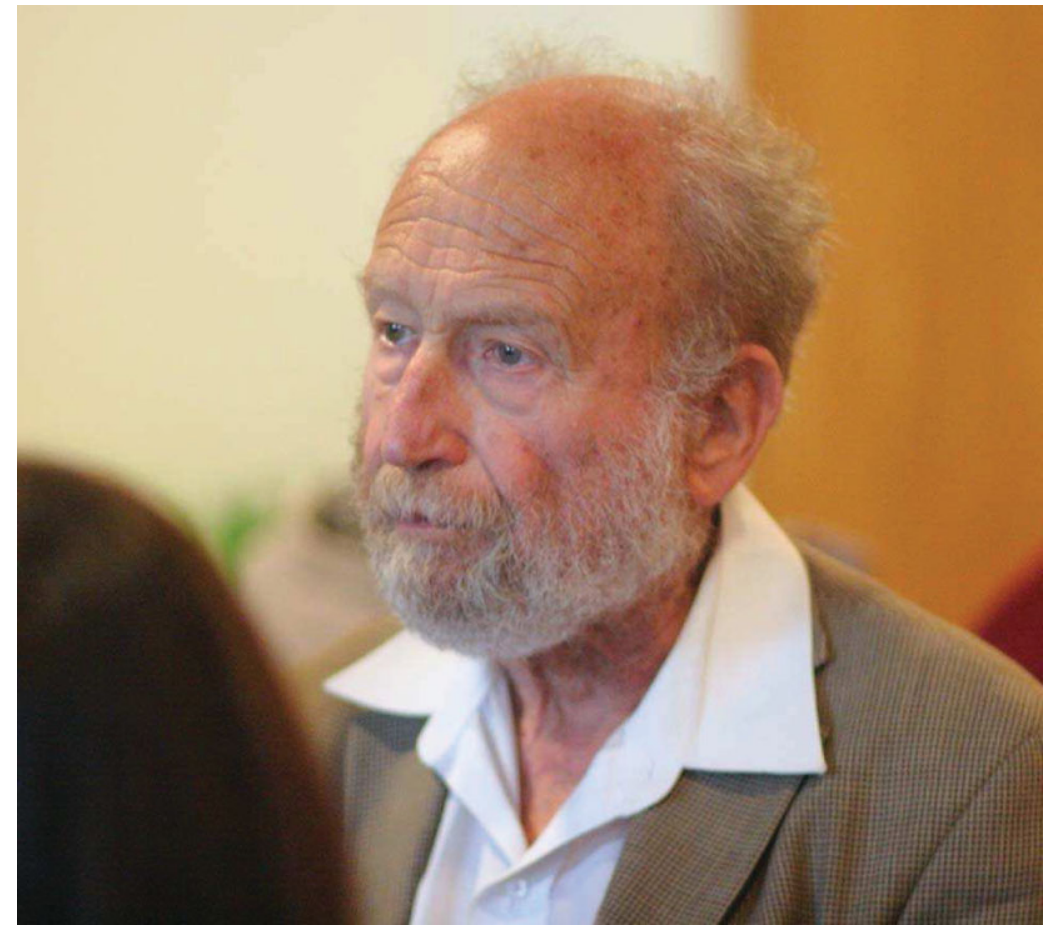

George Wallerstein

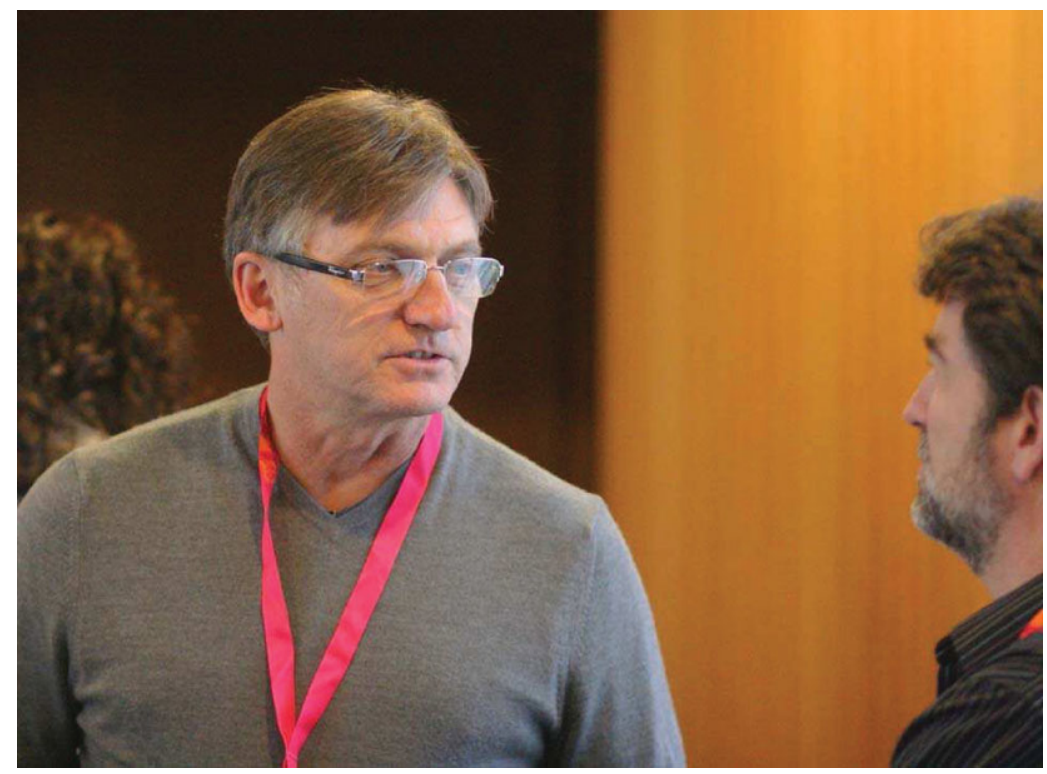

Verne Smith and Evan Skillman 


\title{
Observations of Lithium in red giant stars
}

\author{
Verne V. Smith ${ }^{1}$ \\ ${ }^{1}$ National Optical Astronomy Observatory \\ 950 N. Cherry Ave., Tucson, AZ 85719 USA \\ email: vsmith@noao.edu
}

\begin{abstract}
Connections between observations of the lithium abundance in various types of red giants and stellar evolution are discussed here. The emphasis is on three main topics; 1) the depletion of $\mathrm{Li}$ as stars ascend the red giant branch for the first time, 2) the synthesis of ${ }^{7} \mathrm{Li}$ in luminous and massive asymptotic giant branch stars via the mechanism of hot-bottom burning, and 3) the possible multiple sources of excess $\mathrm{Li}$ abundances found in a tiny fraction of various types of $\mathrm{G}$ and $\mathrm{K}$ giants.
\end{abstract}

Keywords. Stars: abundances, late-type

\section{Introduction}

The connection between lithium and evolution along the red giant branch has a long history, going back to early work by Bonsack (1959), who noted that lithium abundances declined towards later spectral types, which was interpreted as astration of $\mathrm{Li}$ in deepening convective envelopes in cooler stars. A quantitative analysis and comparison with models of the effect of red giant evolution on lithium abundances was carried out by Wallerstein (1966), who was able to identify the Li I $\lambda 6707 \AA$ line in both components of the double-lined spectroscopic binary Capella. Wallerstein found that the more luminous $\mathrm{G}$-giant in the system had 60 times less Li than the less-evolved F-star component: the larger convective envelope of the $\mathrm{G}$-giant had diluted its $\mathrm{Li}$. The factor of 60 in dilution was in excellent agreement with predictions from stellar models by Iben (1965). Lithium had now become an important quantitative test of stellar evolution.

The simple evolution of the dilution of lithium from main sequence to red giant branch is not the whole story of "Lithium in Red Giants", however, due to the complex evolution of stars, initially up the first ascent of the red giant branch (which is referred to here as RGB), followed by evolution along the asymptotic giant branch (AGB), with both destruction and possible synthesis of lithium taking place during different evolutionary phases.

This short summary of lithium in red giants is divided into three sections:

- observations of Li in predominantly RGB stars, where mostly dilution/destruction occurs.

- the behavior of Li in AGB stars, where both destruction, as well as synthesis of ${ }^{7} \mathrm{Li}$ (via so-called "hot bottom burning") can take place.

- the nature of Li-rich $\mathrm{G}$ - and $\mathrm{K}$-giant stars, where only dilution is nominally expected.

\section{Lithium in first-ascent red giants}

Due to the deepening convective envelope that occurs as stars evolve from the main sequence to the RGB, it is expected that the lithium abundance in RGB stars, in general, will be much lower than that observed in main sequence stars that have not destroyed their surface Li. By and large, this simple prediction has been born out by surveys of red 
giants; two early quantitative abundance surveys contained 81 stars of spectral types G, K, and M by Lambert, Dominy, \& Sivertson (1980) and Luck \& Lambert (1982).

A more extensive survey of $644 \mathrm{G}$ and $\mathrm{K}$ giants was presented by Brown et al. (1989) and their abundance results are summarized in histogram form in Figure 1. In Figure 1 the number of stars is plotted versus the lithium abundance, where $\mathrm{A}(\mathrm{Li})$ is the standard spectroscopic definition of $\mathrm{A}(\mathrm{x})=\log [\mathrm{N}(\mathrm{x}) / \mathrm{N}(\mathrm{H})]+12.0$. The arrow indicates the solar system meteoritic abundance $(\mathrm{A}(\mathrm{Li})=3.25)$ and the shift of the red giants to lower abundances is clear. Below abundances of about +0.50 in $\mathrm{A}(\mathrm{Li})$, the histogram is dominated by upper limits (that is, non-detections of the Li I $\lambda 6707 \AA$ line), so the real abundance distribution tails off to even lower Li abundances. There are two main points to take from Figure 1, the first of which is that the overall abundance distribution contains a large fraction of red giants with lower $\mathrm{Li}$ abundances than were predicted from standard convective dilution, thus suggesting that extra-mixing processes on the RGB removed more Li from the surface. The second point is that Brown et al. (1989) were able to quantify the percentage of $\mathrm{G}$ and $\mathrm{K}$ giants which contain more Li than predicted (giants with $\mathrm{A}(\mathrm{Li}) \geqslant 1.5$ are considered to be "Li rich"): this small number of Li-rich giants can be seen in Figure 1. The number of Li-rich giants is roughly $\sim 1 \%$ of the total (this topic will be discussed further in Section 4).

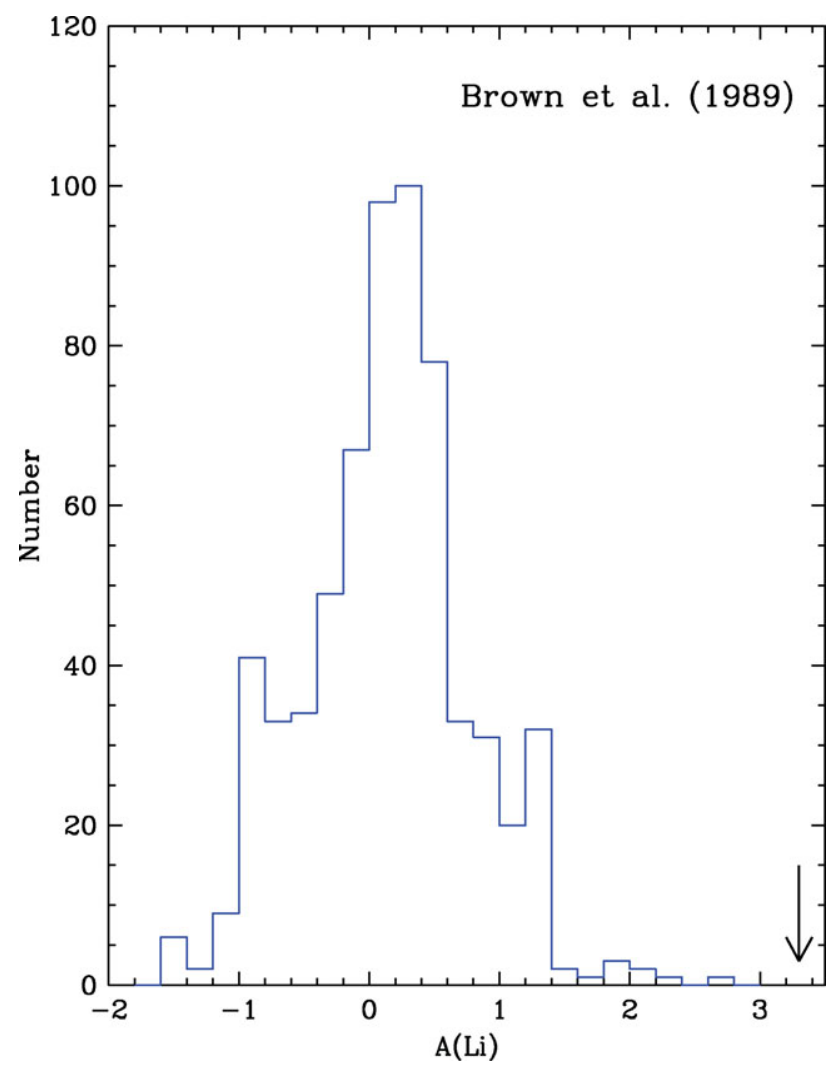

Figure 1. The distribution of lithium abundances in $644 \mathrm{G}$ and $\mathrm{K}$ giants from Brown et al. (1989). Note that non-detections dominate the statistics below $\mathrm{A}(\mathrm{Li}) \sim+0.5$. The distribution of abundances suggests that extra-mixing and more astration of Li occurs on the RGB, with about $\sim 1 \%$ of the giants being "Li rich". The arrow indicates the solar system value of $\mathrm{A}(\mathrm{Li})$. 
Gratton et al. (2000) conducted a survey of field metal-poor giants to study mixing, with lithium being included as one of their tests. One of the advantages in the Gratton et al. study, was that the luminosities of their sample giants could be estimated reasonably well, so that $\mathrm{A}(\mathrm{Li})$ could be investigated as a function of position on the RGB. The top panel of Figure 2 summarizes the results from Gratton et al. (2000), with A(Li) shown versus luminosity. The vertical dashed lines indicate the approximate luminosity of two important locations in an HR diagram; the first one shows the approximate location of the base of the RGB (at $\left.\log \left(\mathrm{L} / \mathrm{L}_{\odot}\right) \sim+0.8\right)$, while the second illustrates roughly where the RGB luminosity "bump" falls in low-mass low-metallicity giants. The dramatic drop in the $\mathrm{Li}$ abundance at the beginning of evolution up the RGB stands out clearly, with the abundance of lithium then remaining roughly constant, until another drop at the RGB bump. Displayed as in Figure 2, the evolution of the lithium abundance in most RGB stars seems reasonably well-defined.

Recent work on Li depletion along the RGB has focused on the globular cluster NGC6397, with studies by Korn et al. (2007) or Lind et al. (2009). In the case of a globular cluster, such as NGC6397, the advantage is that the luminosity (or put another way, the position on the RGB) is very well determined. The bottom panel of Figure 2 shows results from the Korn et al. (2007) study, where their results for 18 stars are averaged into 4 distinct evolutionary points: in increasing luminosity, the subgiant branch, the base of the RGB, the lower RGB, and the upper RGB. The overall behavior of $\mathrm{A}(\mathrm{Li})$ in the NGC6397 stars closely matches that for the metal-poor low-mass field giants from Gratton et al. (2000-the top panel of Figure 2). The recent work on NGC6397 (Korn et al. 2007; Lind et al. 2009) includes diffusion in their modelling and the results between Li depletion predicted by theory and what is observed is quite good.

Progress continues to be made using lithium to probe RGB evolution, e.g. Lagarde et al. (2009, this symposium) presents Li abundances in a large sample of field red giants, with luminosities derived from Hipparcos parallaxes, which were not available to Brown et al. (1989). It can be stated that our understanding of the behavior of Li abundances along the RGB is much better than it was compared to just a few years ago.

\section{Lithium in hot-bottom burning asymptotic giant branch stars}

Asymptotic Giant Branch stars are well-known producers of ${ }^{12} \mathrm{C}$, with this primary carbon-12 produced during phases of shell- ${ }^{4} \mathrm{He}$-burning in thermal pulses, which drive convective mixing of ${ }^{12} \mathrm{C}$-rich material to the stellar surface. This mixing results in the evolutionary sequence running from the oxygen-rich $(\mathrm{C} / \mathrm{O} \leqslant 1)$ M-type giants to the carbon stars $(\mathrm{C} / \mathrm{O} \geqslant 1)$. In addition to the production of ${ }^{12} \mathrm{C}$, the mixing between Heburning shell and $\mathrm{H}$-rich envelope results in the release of free neutrons via the chain ${ }^{12} \mathrm{C}(\mathrm{p}, \gamma){ }^{13} \mathrm{~N}\left(\beta^{+}, \nu\right){ }^{13} \mathrm{C}(\alpha, \mathrm{n}){ }^{16} \mathrm{O}$. These neutrons drive the s-process and synthesize the neutron-rich nuclei heavier than $\mathrm{Fe}$ (such as $\mathrm{Zr}$ or Ba).

The synthesis of ${ }^{12} \mathrm{C}$ and the s-process elements, followed by dredge-up (the so-called "third dredge-up") to produce C- and s-process-rich AGB stars, occurs in a large fraction of AGB stars from $\mathrm{M} \sim 1-8 \mathrm{M}_{\odot}$, with the exact mass limits depending on stellar metallicity. Another process, referred to as "hot-bottom burning" (HBB), first studied in model stars by Scalo, Despain, \& Ulrich (1975), occurs in the more massive AGB stars (say M $\geqslant 4 \mathrm{M}_{\odot}$ ) and can result in the production of ${ }^{7} \mathrm{Li}$ via the Cameron-Fowler mechanism (Cameron \& Fowler 1971).

As a result of $\mathrm{HBB}$, very Li-rich AGB stars can be created and observations pointed in this direction long before any understanding of internal nucleosynthesis in AGB stars. McKellar (1940) observed a very strong Li I $\lambda 6707 \AA$ line in the carbon star WZ Cas and 
suggested that this star had "an unusually high abundance of lithium". A survey of 30 disk carbon stars was conducted by Torres-Peimbert \& Wallerstein (1966), who found some 16 of these stars to exhibit substantial Li I lines. The phenomenon of Li-rich AGB stars was extended to the S-stars (AGB stars with enhanced s-process and carbon-12 abundances, but with C/O still less than 1.0) with Keenan's (1967) detection of a strong Li I line in T Sgr.

Quantitative lithium abundance analyses of S stars began with Boesgaard (1970), who found very large abundances in a few S stars (including $\mathrm{T}$ Sgr), with values as large as $\mathrm{A}(\mathrm{Li}) \sim 4.0$ ( $\sim 10 \mathrm{x}$ the solar system abundance). Observationally measured luminosities of HBB Li-rich AGB stars was placed on a firmer footing by observations of such stars in the Magellanic Clouds (Smith \& Lambert 1989; 1990; Plez et al. 1993; Smith et al. 1995), since the distances to the LMC and SMC are quite well known. More recently, attention has turned back to the Milky Way, with work by Garcia-Hernandez et al. (2007),

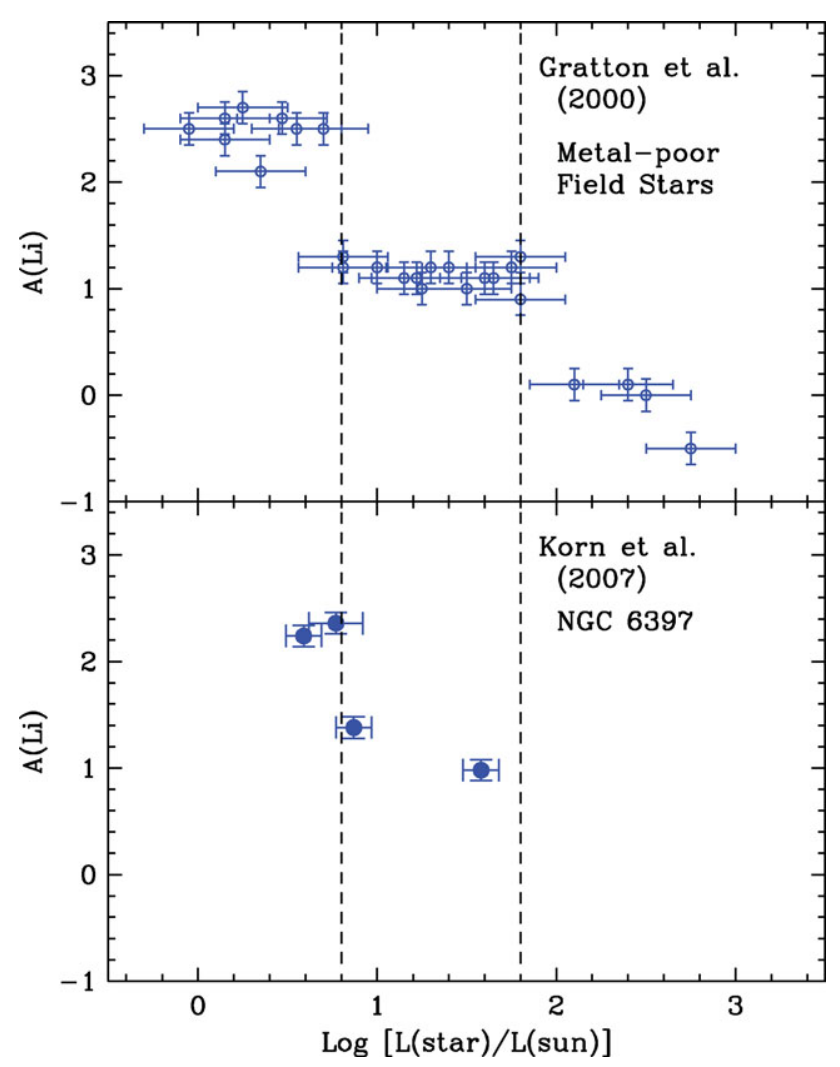

Figure 2. The top panel shows results from Gratton et al. (2000) lithium abundances in metal-poor low-mass field stars plotted versus luminosity. The vertical dashed lines mark the approximate luminosities of the base of the RGB (left line) and the RGB luminosity bump (right line). The precipitous drop on $\mathrm{A}(\mathrm{Li})$ as stars evolve onto the RGB is clear, with another decline near the location of the RGB bump. The bottom panel illustrates lithium abundances in stellar members of the globular cluster NGC6397 from Korn et al. (2007). The 4 points are averages from a sample of 18 stars, with the points representing stars on the subgiant branch, the base of the RGB, the lower RGB, and upper RGB. The behavior of the lithium abundance in the lowmass low-metallicity stars in NGC6397 is similar to those in the field-star sample from Gratton et al. (2000). 
who determined Li abundances (along with other elements, including the s-process) in luminous Galactic AGB stars. A convenient way to view the combined lithium abundances in Galactic, LMC, and SMC luminous AGB stars is illustrated in Figure 3, where $\mathrm{A}(\mathrm{Li})$ is plotted versus pulsational period (all of these cool stars are long period variables). The overall trend is for the period to increase with increasing luminosity, so this figure shows the somewhat abrupt increase in lithium for periods greater than about 300400 days. This figure also demonstrates that although ${ }^{7} \mathrm{Li}$ can be produced in amounts larger than that found in the current disk ISM, it can also be destroyed, so there is a wide distribution of lithium abundances in the luminous AGB stars; the exact net amount of ${ }^{7} \mathrm{Li}$ that might be contributed by these types of stars to chemical evolution remains uncertain, primarily due to uncertainties in how mass loss occurs along the AGB. Stellar models of HBB along the AGB by Sackmann \& Boothroyd (1992) can produce the upper envelope of $\mathrm{A}(\mathrm{Li})$ observed in Figure 3 for stellar masses of $4-6 \mathrm{M}_{\odot}$ at $\mathrm{M}_{\mathrm{Bol}} \sim-6.2$ to -6.8 .

One interesting consequence of $\mathrm{HBB}$ in $\mathrm{AGB}$ stars is the conversion of primary ${ }^{12} \mathrm{C}$ (sometimes lots of it) into ${ }^{14} \mathrm{~N}$ via the $\mathrm{CN}$-cycle taking place within the deep convective envelope itself. The drop in ${ }^{12} \mathrm{C}$ abundance can result in the surface $\mathrm{C} / \mathrm{O}$ ratio falling below 1.0, and the transformation of a carbon star back into an oxygen-rich star (in this case, since it would be s-process enriched star, it would be an S-star). This effect

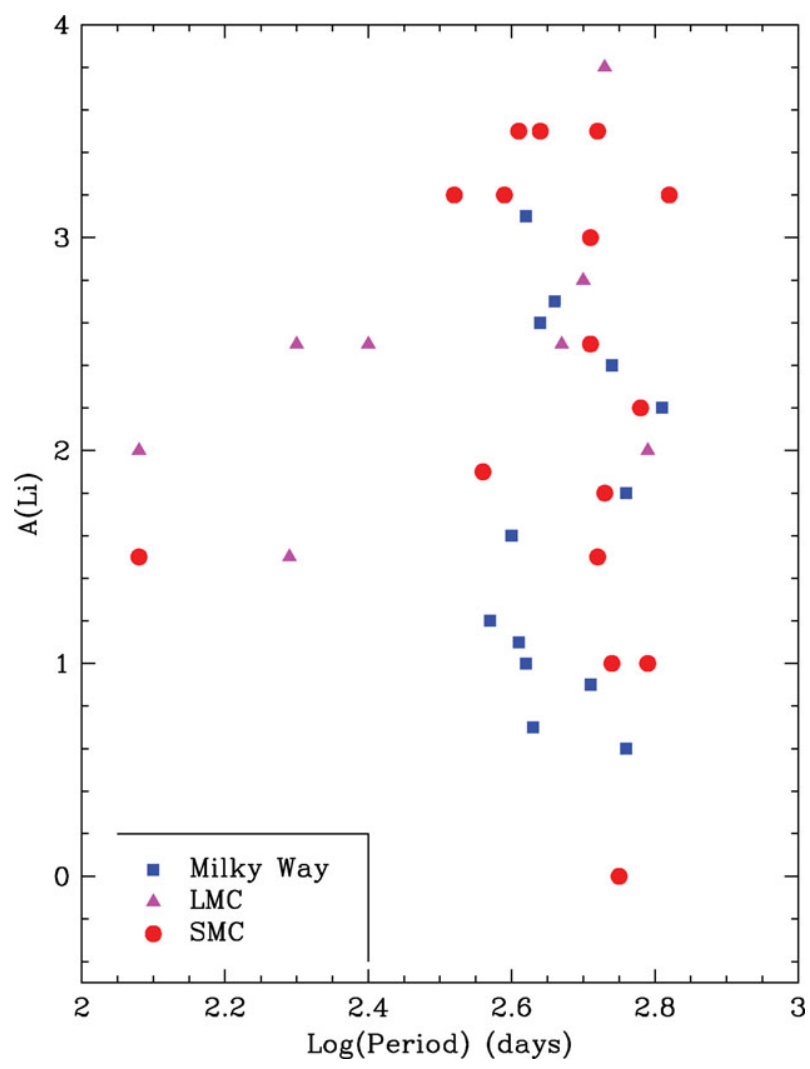

Figure 3. Lithium abundances versus pulsational period for luminous Galactic AGB stars from Garcia-Hernandez et al. (2007; filled blue squares) and for luminous SMC and LMC AGB stars from Smith et al. (1995); filled red circles for the SMC and filled magenta triangles for the LMC. The upper envelope of $\mathrm{A}(\mathrm{Li})$ is predicted by models of HBB in AGB stars by Sackmann \& Boothroyd (1992). 
is illustrated in Figure 4 for the LMC, where the C-star luminosity function from Iben (1981) is shown compared to the location, in luminosity space (with luminosity indicated by $\mathrm{M}_{\mathrm{Bol}}$ ), of the Li-rich S-stars. At the bolometric luminosity at which the number of C-stars drops to zero, the appearance of Li-rich S-stars takes place, with the lithium indicating the onset of $\mathrm{HBB}$. With enough conversion of ${ }^{12} \mathrm{C}$ to ${ }^{14} \mathrm{~N}$ in the hot envelopes of these stars, the $\mathrm{C} / \mathrm{O}$ ratio is driven back down below unity and former carbon stars transform to S-stars.

\section{The mysterious Lithium-rich red giants}

As discussed in Section 2, stellar evolutionary models predict that lithium will be depleted during the first ascent of the RGB, with the amount of this depletion being 1 to 2 orders of magnitude. It was not expected that there would be significant sources of $\mathrm{Li}$ in stars evolving along the RGB, until the possible onset of $\mathrm{HBB}$ in massive, luminous AGB stars (the subject of Section 3). However, the discovery of an unexpectedly high lithium abundance $(\mathrm{A}(\mathrm{Li})=2.7)$ in the K-giant HD112127 by Wallerstein \& Sneden

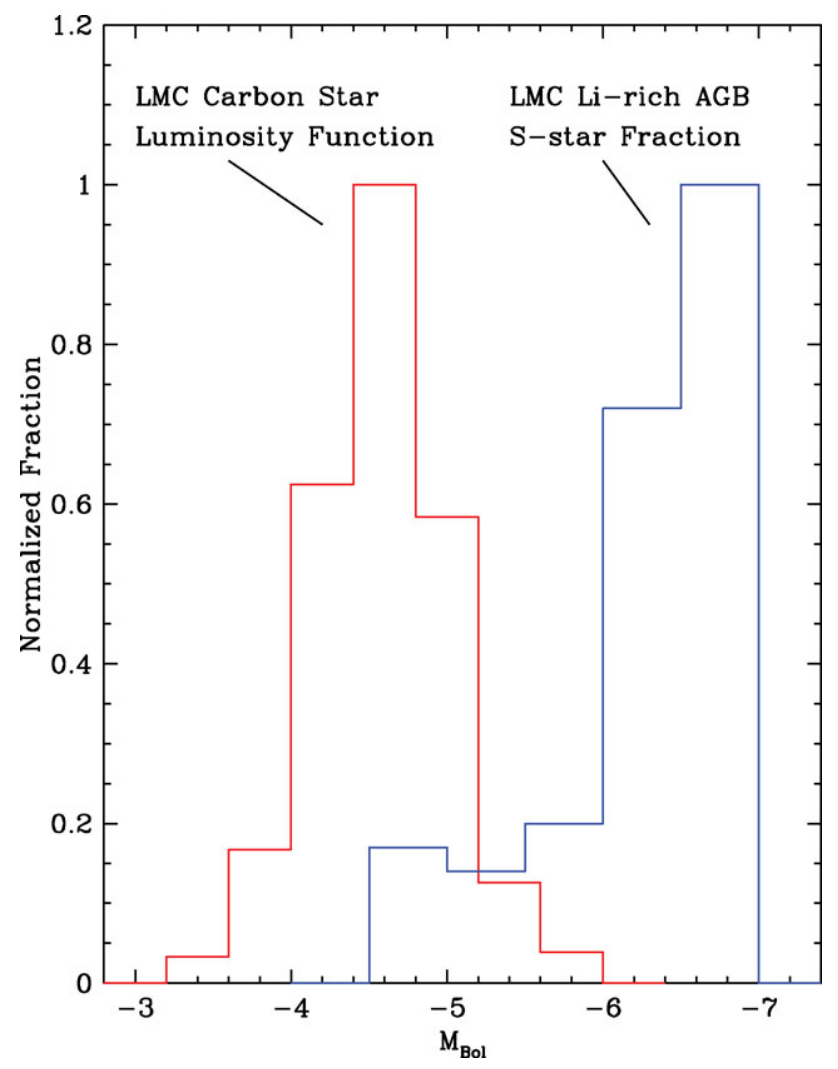

Figure 4. The carbon-star luminosity function for the LMC from Iben (1981) is shown as the lefthand histogram. On the right is plotted the fraction of AGB S-stars that are Li-rich in the LMC (Smith et al. 1995). Note that the fraction of Li-rich AGB stars with $\mathrm{C} / \mathrm{O} \geqslant 1.0$ increases at about the same luminosity that the C-star luminosity function falls to zero; this transition between the two distributions represents the onset of hot bottom burning, the production of lithium and the conversion of carbon stars to luminous S-stars. 
(1982) demonstrated that the behavior of Li in RGB stars was not simple. The subsequent survey by Brown et al. (1989-see Figure 1) showed that about $1 \%$ of $\mathrm{G}$ and $\mathrm{K}$ giants exhibit excess $\mathrm{Li}$ abundances (i.e, $\mathrm{A}(\mathrm{Li}) \geqslant 1.5$ ).

Continuing studies found additional examples of Li-rich RGB stars, with the interesting observation by Fekel \& Balachandran (1993) that a significant fraction of the Li-rich giants are rapidly rotating (with vsini $\geqslant 8 \mathrm{~km}-\mathrm{s}^{-1}$ ). In addition, de la Reza et al. (1996) found that these peculiar giants were surrounded by spatially extended, faint dust shells, as revealed in IRAS colors. Charbonnel \& Balachandran (2000) found that the so-called "super Li-rich" giants (with $\mathrm{A}(\mathrm{Li}) \geqslant 3.3$ ) tended to be found in a specific location of the H-R Diagram, near the luminosity bump at $\log \left(\mathrm{L} / \mathrm{L}_{\odot}\right) \sim 1.4-1.9$ and $\mathrm{T}_{\text {eff }} \sim 4450-4600 \mathrm{~K}$. The combination of rapid rotation, dust shells which suggest mass-loss episodes, and location in a single part of the HR Diagram has led to a picture in which the excess $\mathrm{Li}$ has been produced within the RGB star (via the Cameron Fowler mechanism) and carried to the surface. This dredge-up episode also carried angular momentum from the more rapidly rotating interior and drove a mass loss event. The exact physical mechanism remains elusive, but may be the only viable hypothesis to explain the location of the super Li-rich giants near the luminosity bump.

In addition to the Li-rich RGB stars found in the disk, 3 Li-rich giants have been found in globular clusters; one each in the clusters M5 (Carney et al. 1998), NGC362 (Smith et al. 1999), and M3 (Kraft et al. 1999). Since the globular clusters have well-defined stellar populations and distances, it is straightforward to place these stars in an H-R Diagram. Figure 5 summarizes the locations of the Li-rich G and K giants in a Log L - $\mathrm{T}_{\text {eff }}$ plane and it is immediately clear that the super Li-rich giants (filled circles) are in a distinctly different evolutionary state than the Li-rich globular cluster giants, which turn out to be AGB, or even post-AGB stars. Since the globular cluster AGB stars are not massive enough to have undergone HBB, the source of the lithium must be from a different mechanism. It is also worth noting that there is no evidence of rapid rotation in the 3 Li-rich globular cluster giants. One is tempted to conclude that different physical mechanisms are driving the mixing (if it is internal mixing) in these two classes of Li-rich giants.

Finally in Figure 5, we note the filled squares which represent rapidly rotating Li-rich $\mathrm{G}$ and $\mathrm{K}$ giants found by Carlberg et al. (2009b), and which extend to luminosities well below the luminosity bump. Carlberg et al. (2009b) speculate that these objects may result from the ingestion of closely orbiting large planets or brown dwarfs as the parent star evolves up the RGB. Simulations of the future evolution of known star-hot Jupiter systems by Carlberg et al. (2009a) suggest that such ingestion will occur, typically near the base of the RGB, and would result in an increase in the stellar surface Li abundance, as well as spin-up of the convective envelope.

With such a variety of low-mass giants exhibiting enhanced lithium abundances spread across the H-R Diagram, it may well be that the phenomenon of the Li-rich G and $\mathrm{K}$ giants is caused by a number of different mechanisms. Observers and modelers have much to sort out in this particular area.

\section{Acknowledgements}

I wish to thank the meeting organizers for inviting me to present this review of a truly fascinating and rich topic, involving lithium and stellar evolution. I would also like to note that of the 3 main issues that were covered here, George Wallerstein (one of the meeting attendees) played key roles in the early stages of each one of them: he is an observational pioneer in this area. Since GW was my thesis advisor, I would also like to 


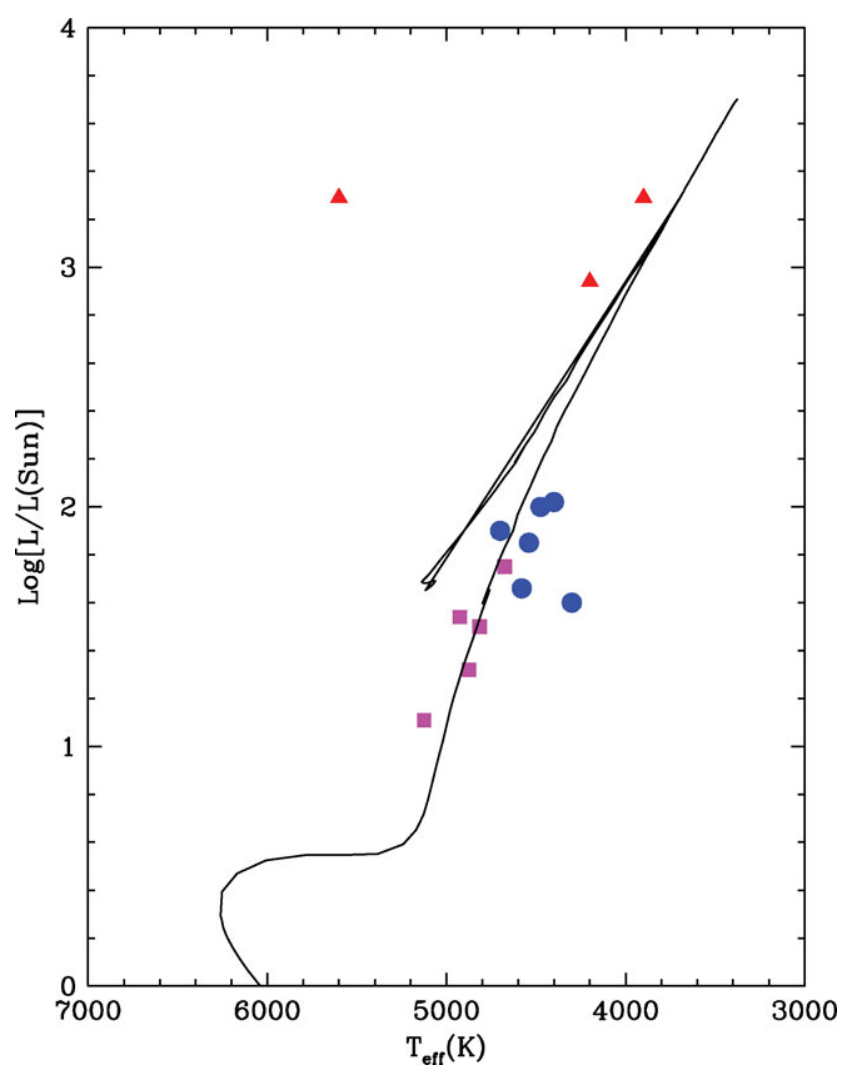

Figure 5. Luminosities and effective temperatures for samples of K-giants with enhanced lithium abundances. The filled triangles are stars in globular clusters from Carney et al. (1998) for M5, Smith et al. (1999) for NGC362, and Kraft et al. (1999) for M3. Filled circles are field Kgiants discussed in Kumar \& Reddy (2009), while the filled squares are rapidly-rotating Li-rich giants noted in Carlberg et al. (2009b). This type of diagram suggests that the "lithium-rich K-giant" phenomenon occurs over a wide evolutionary range and may be due to different processes, depending on the evolutionary state of the red giant. The solid line is a sample isochrone for a metallicity of -0.5 and an age of $9 \mathrm{Gyr}$ (or a giant mass of about $1.3 \mathrm{M}_{\odot}$ ), not very different from the plotted giants; this stellar evolutionary track indicates that the Li-rich giants are found from near the base of the red giant branch to well along and past the AGB.

take this opportunity to thank him for introducing me, in his own unique style, to each of these topics and providing a background that stressed the key role of spectroscopy.

\section{References}

Boesgaard, A. M. 1970, ApJ, 161, 1003

Bonsack, W. K. 1959, ApJ, 130, 843

Brown, J. A., Sneden, C., Lambert, D. L., \& Dutchover, E., Jr. 1989, ApJS, 71, 293

Cameron, A. G. W. \& Fowler, W. A. 1971, ApJ, 164, 111

Carlberg, J. K., Majewski, S. R., \& Arras, P. 2009a, ApJ, 700, 832

Carlberg, J. K., Majewski, S. R., Smith, V. V., Cunha, K., Patterson, R. J., Bizyaev, D., Arras, P., \& Rood, R. T. 2009b, in: K. Cunha, M. Spite, \& B. Barbuy (eds.), Chemical Abundances in the Universe: Connecting First Stars to Planets (Cambridge: Cambridge University Press), p. 408

Carney, B. W., Fry, A. M., \& Gonzalez, G. 1998, AJ, 116, 2984

Charbonnel, C. \& Balachandran, S. 2000, A\&AA, 359, 563 
de La Reza, R., Drake, N. A., \& da Silva, L. 1996, ApJ (Letters), 456, 456

Fekel, F. C. \& Balachandran, S. 1993, ApJ, 403, 708

Garcia-Hernandez, D. A., Garcia-Lario, P., Plez, B., Manchando, A., D’Antona, F., Lub, J., \& Habing, H. 2007, A\&BA, 462, 711

Gratton, R. G., Sneden, C., Carretta, E., \& Bragaglia, A. 2000, A\& A, 354, 169

Iben, I., Jr. 1965, ApJ, 142, 1447

Iben, I., Jr. 1981, $A p J, 243,987$

Keenan, P. C. $1967, A J, 72,808$

Korn, A. J., Grundahl, F., Richard, O., Mashonkina, L., Barklem, P. S., Collet, R., Gustafsson, B., \& Piskunov, N. 2007, ApJ, 671, 402

Kraft, R. P., Peterson, R. C., Guhathakurta, P., Sneden, C., Fulbright, J. P., \& Langer, G. E. 1999, ApJ, 518, L53

Kumar, Y. B. \& Reddy, B. E. 2009, ApJ (Letters), 703, 46

Lambert, D. L., Dominy, J. F., \& Sivertsen, S. 1980, ApJ, 235, 114

Lind, K., Primas, F., Charbonnel, C., Grundahl, F., \& Asplund, M. 2009, Aछ A, 503, 545

Luck, R. E. \& Lambert, D. L. 1982, ApJ, 256, 189

McKellar, A. 1940, PASP, 52, 407

Plez, B., Smith, V. V., \& Lambert, D. L. 1993, ApJ, 418, 812

Sackmann, I.-J. \& Boothroyd, A. I. 1992, ApJ, 392, 71

Scalo, J. M., Despain, K. H., \& Ulrich, R. K. 1975, ApJ, 196, 805

Smith, V. V. \& Lambert, D. L. 1989, ApJ (Letters), 345, 75

Smith, V. V. \& Lambert, D. L. 1990, ApJ (Letters), 361, 69

Smith, V. V., Plez, B., Lambert, D. L., \& Lubowich, D. A. 1995, ApJ, 441, 735

Smith, V. V., Shetrone, M. D., \& Keane, M. J. 1999, ApJ (Letters), 516, 73

Torres-Peimbert, S. \& Wallerstein, G. 1966, ApJ, 146, 724

Wallerstein, G. 1966, ApJ, 143, 823

Wallerstein, G. \& Sneden, C. 1982, ApJ, 255, 577 